\title{
MAGNETIC FIELDS IN COOLING FLOW CLUSTERS
}

\author{
R. A. PERLEY \\ National Radio Astronomy Observatory \\ P.O. Box O \\ Socorro, N.M. 87801 \\ U.S.A.
}

\begin{abstract}
Faraday rotation measurements of the luminous radio galaxies $3 \mathrm{C} 295$, Cygnus A, and Hydra A, all of which are associated with $\mathrm{cD}$ galaxies located in massive cooling flow clusters, show very high rotation measures - typically a few thousand $\mathrm{rad} \mathrm{m}^{-2}$, exceeding $20,000 \mathrm{rad} \mathrm{m}^{-2}$ for $3 \mathrm{C} 295$. The RMs vary over large ranges and with different scales. The RMs are both positive and negative, implying reversals of the magnetic field - the variations cannot be due to density changes alone. The RMs originate in magnetized cluster gas, but it is unclear what fraction is due to a coccoon surrounding the radio source. If from the cluster as a whole, the magnetic field is $B \sim 2 \sqrt{N} \mu \mathrm{G}$, where $N$ is the number of cells along the line of sight. A good case can be made for an enhanced magnetic field localized at or near the surface of the radio source, accounting for a significant fraction of the observed RM.
\end{abstract}

\section{Introduction}

$\mathrm{X}$-ray observations have indicated that many galaxy clusters must have a slow infall of gas, which evidently cools as it settles to the center. However, the normal conductivity of this gas should reduce the temperature gradient sufficiently to prevent the flow. The existence of these 'cooling flows' requires suppression of the conductivity, and the most obvious way is through the presence of a magnetic field. Observations indicating the presence, or absence of such fields are clearly important.

One straightforward, although painstaking method of unambiguously detecting magnetic fields is through measurement of the Faraday rotation. If a source of linearly polarized radiation lies behind, or within a cluster, the plane of polarization of the observed radiation will be rotated by an amount given by $\Delta \theta=R M \lambda^{2}$, where $R M$ is the Rotation Measure, usually expressed in $\mathrm{rad} \mathrm{m}^{-2}$. The $\mathrm{RM}$ is related to the magnetic field through $R M=0.81 \int n_{e} B d L$, where $n_{e}$ is the electron density in $\mathrm{cm}^{-3}, B$ is the magnetic field in $\mu \mathrm{G}$, and the pathlength $L$ is expressed in pc. If the magnetoionic medium, or Faraday screen, lies entirely outside the source of radiation, and the source is observed with sufficient resolution to resolve out transverse variations in the structure of the screen, then a plot of the position angle

R. Beck et al. (eds.), Galactic and Intergalactic Magnetic Fields, 463-468.

C) 1990 IAU. Printed in the Netherlands. 
vs. $\lambda^{2}$ will be exactly linear with a slope equal to the RM. Non-linearities indicate either insufficient resolution, or thermal gas within the emitting source. These alternatives are often difficult to separate. Measurement of the RM, combined with knowledge of the distribution and extent of the thermal gas allows a direct measure of the magnetic field in the screen.

Over the past few years, many extragalactic radio sources have had their RM distributions mapped. For most sources, the RMs are small, generally less than $50 \mathrm{rad} \mathrm{m}^{-2}$. But for some sources, all of which are identified with clusters of galaxies, the RMs are very much higher. At the N.R.A.O. in Socorro N.M., two separate approaches are being attempted to explore the range of the rotation measure phenomenon. One is by Frazer Owen and his graduate student Jing Ping Ge. They are measuring the RMs in weak radio sources found in the centers of massive cooling flow clusters. The second approach is being taken by myself and my students Chris Carilli and Greg Taylor. Our approach is to map the RMs of sources known a priori through observations at lower resolutions at high frequencies to have anomalously high rotation measures. In this contribution, I will summarize the results of our work.

\section{Low-Power Radio Sources in Dense Clusters}

Frazer Owen and Jing Ping Ge have selected a sample of 7 lower-power radio sources identified with $\mathrm{cD}$ galaxies at the center of dense cooling flow clusters. The following table shows the sources, their characteristics, and the status of the project. Some details follow the table.

\begin{tabular}{|l|r|l|c|l|}
\hline Source & $M_{\odot}$ & $\mathrm{z}$ & $\log P_{1.4}$ & \multicolumn{1}{c|}{ Status } \\
\hline 3C338 & 120 & .030 & 25.13 & $|R M|<1000 \mathrm{rad} \mathrm{m}^{-2}$ \\
$\mathrm{~A} 2151$ & 115 & .037 & & Data taken, no reduction \\
$\mathrm{A} 2052$ & 125 & .035 & 25.49 & $|R M|<500 \mathrm{rad} \mathrm{m}^{-2}$ \\
$\mathrm{~A} 1795$ & 300 & .06 & 25.23 & $R M<4000 \mathrm{rad} \mathrm{m}^{-2}$, all positive \\
$\mathrm{A} 133$ & 90 & .06 & & Too weak \\
$3 \mathrm{C} 84$ & 300 & .018 & 25.43 & Long shot - no results to date \\
$\mathrm{M} 87$ & 10 & .0034 & 25.03 & Galaxy has RMs to 8500 rad $\mathrm{m}^{-2}$ \\
\hline
\end{tabular}

The physical sizes of most of these sources are rather small - many lie entirely within the host galaxy. Thus, the measured RMs could be dominated by galaxian fields rather than intracluster fields. The source $3 \mathrm{C} 338$ is quite large, and has RMs exceeding $1000 \mathrm{rad} \mathrm{m}^{-2}$ in a few regions clearly outside the galaxy. For the lobes of M87, which also extend beyond the galaxy, the situation is less clear - the relevant data have not yet been reduced, although it is clear that the RMs are unlikely to exceed $1000 \mathrm{rad} \mathrm{m}^{-2}$. Strong depolarization is noted in the inner $2 \mathrm{kpc}$ of A1795, but this is certainly associated with strong emission line gas detected from the same area. A2052 is not a double, but appears (in the radio) more akin to a faceon spiral, with circumferential magnetic fields. Data taking and reduction is still 
proceeding for all of these sources. Comprehensive results await completion of this work.

\section{High-Power Radio Sources in Dense Clusters}

Our approach to the problem has been driven by our original observations of the prominent radio galaxy Cygnus $\mathrm{A}$. This object is located at the center of a dense $\mathrm{X}$-ray emitting cluster, and shows an extremely high RM. To assist in identifying the origin of the required fields, we are engaged in mapping the RMs of other radio galaxies of similar radio luminosity and environment. The objects were selected using the list of Kato et al. (1987), which gives a compilation of extragalactic radio sources with very high Faraday rotation. This list has been recently updated by Aizu et al., (this conference), which lists 18 extragalactic objects with unusually high RMs. Of these 18 , there are 5 sources with RMs exceeding $1000 \mathrm{rad} \mathrm{m}^{-2}$. Of these, three are compact steep spectrum objects, too small to be resolved by the VLA. The remaining two objects, 3C295 and 3C218 (= Hydra A), have been intensively observed by us to map the RM structure.

These two objects, plus Cygnus A (which is not in either finding list due to its low galactic latitude), share two remarkable properties:

- All three are identified with $\mathrm{cD}$ galaxies in dense cooling flow clusters.

- All three are extremely luminous, with spectral powers at $1400 \mathrm{MHz}$ exceeding $10^{27}$ watt/Hz. From these properties, it seems clear that high RM radio galaxies are identified with high radio luminosity and high local gas density.

\subsection{RM DISTRIBUTIONS FOR LUMINOUS SOURCES}

3.1.1 Cygnus $A$ The detailed results have been published by Dreher et al. (1987). Cygnus $A$ has an extremely high luminosity, but is of rather average size, resulting in very high internal pressures and equipartition fields. For the lobes, $B_{e q} \sim 60 \mu G$. Perusal of the RM distribution (as shown in Dreher et al., (1987) reveals (to me, at least), four different scales of RM changes:

- There is large-scale order. For instance, a large fraction of the Western lobe shows no significant change in the RM on scales of tens of kpc.

- Large scale gradient. In both lobes, but more notably in the Western lobe, there is a large-scale $(>20 \mathrm{kpc}$ ) change in $R M$, running along the axis of the source. The gradient is approximately $100 \mathrm{rad} \mathrm{m}^{-2}$ per $\mathrm{kpc}$. In the Western lobe, the $\mathrm{RM}$ changes from large positive at the Western extremnity to equally large negative values over the length of the lobe. The same gradient is visible in the Eastern lobe, although the paucity of data (due to depolarization which we believe to be external) prevents a firm detection.

- Small scale gradients. There exist rapid changes of RM within each lobe, with gradients exceeding $600 \mathrm{rad} \mathrm{m}^{-2}$ per kpc on scales less than $10 \mathrm{kpc}$. One of the most notable is the RM arc centered on hot spot ' $\mathrm{B}$ ' in the Western lobe. Carilli, Perley and Dreher (1988) have interpreted this as a signature of a bow shock driven into the ICM by the advancing hotspot.

- Discontinuous changes. In a number of locations, there are jumps in RM, of up to $3000 \mathrm{rad} \mathrm{m}^{-2}$. There is no transition visible, so the change occurs within a beamwidth, less than $400 \mathrm{pc}$. 


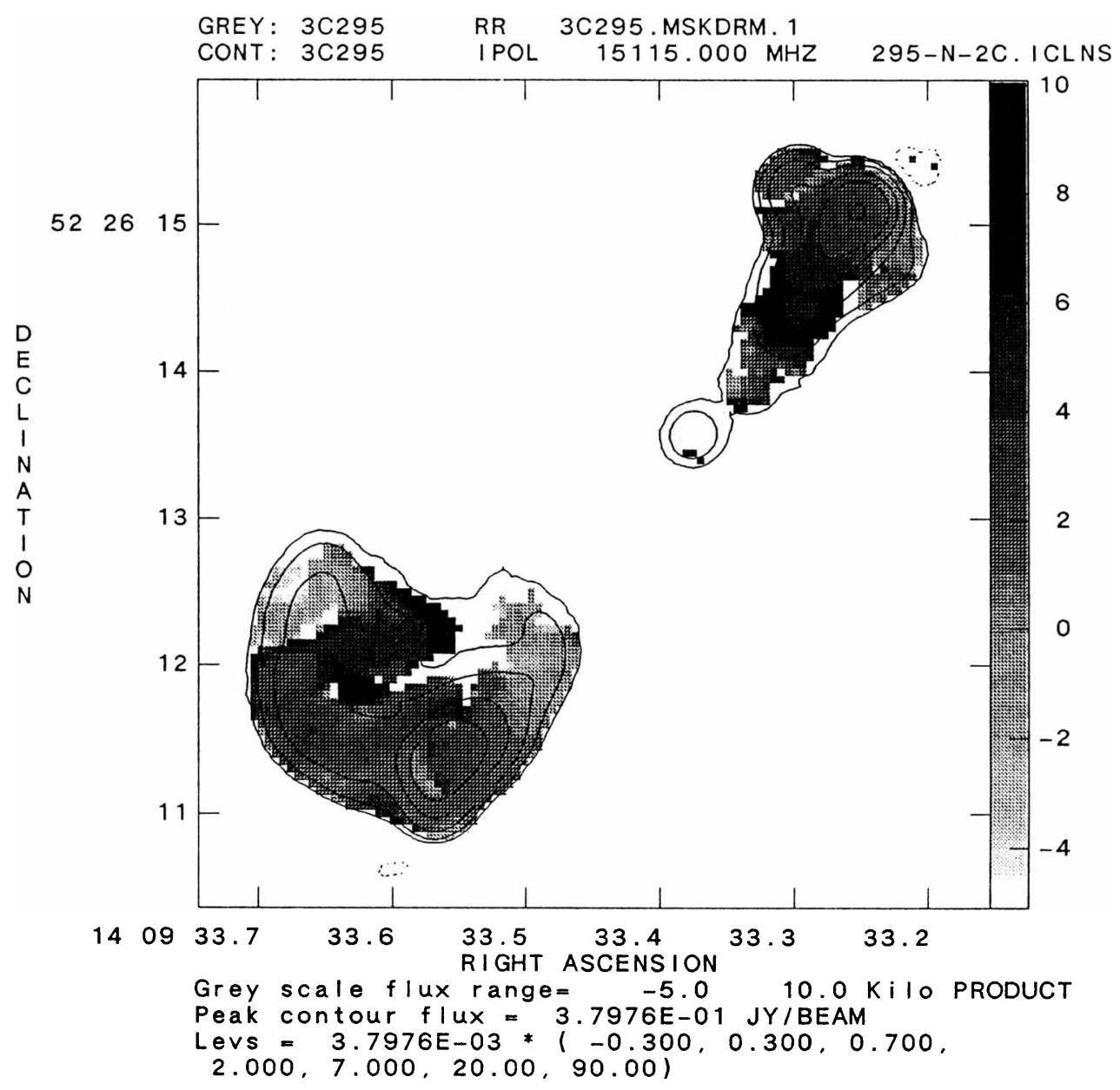

Figure 1. A grey-scale representation of the Rotation Measure in 3C295. The greyscale runs from $-5000 \mathrm{rad} \mathrm{m}^{-2}$ (light) to $10,000 \mathrm{rad} \mathrm{m}^{-2}$ (black). The RMs must be multiplied by two to account for the source redshift. Blanked areas show as pure white in this figure. Note the discontinuous change in RM in the Southern lobe.

3.1.2 3C295 This object was originally chosen because its radio spectrum, radio luminosity, and X-ray characteristics were essentially identical to those of Cygnus A. 3C295 also appears in the lists of Kato et al., and Aizu et al.. It is a radio galaxy of rather small size, less than $20 \mathrm{kpc}$; combined with its extreme radio luminosity, the equipartition fields are extremely high, $\sim 250 \mu G$. The RM image is shown in Fig. 1, in a grey-scale representation. The RM ranges from -10000 to $+10000 \mathrm{rad} \mathrm{m}^{-2}$ - but these must be multiplied by 2 to account for the redshift dependence $(1+z)^{2}$. Note the large-scale order (although the scale is actually 
rather small), and the extraordinary jump in the southern lobe.

It should be noted that both $3 \mathrm{C} 295$ and Cygnus $\mathrm{A}$ are rather small for investigations into the variation of magnetic field with cluster radius, since both objects are significantly smaller than a cluster core radius. Our third object is of such size that these investigations are possible.

3.1.3 Hydra A Hydra A (Taylor et al, 1989), is another very high luminosity radio source, but is of very different morphology than the preceding sources. Although of a luminosity well above the FRI - FRII break, Hydra A is clearly of FRI morphology. It is similar to Wide-Angle Tail sources (WATs), but of significantly higher luminosity. The RMs are again very high, up to $\pm 3000 \mathrm{rad} \mathrm{m}^{-2}$ for the northern lobe. The RMs for the southern lobe are higher, but strong depolarization effects have prevented a measurement. Our new data, being taken at $3.6 \mathrm{~cm}$, are expected to recover the RMs from this region. Perhaps the most interesting aspect of Hydra A is that it extends a total of $250 \mathrm{kpc}$ from the nucleus, giving us an opportunity of measuring the RMs over a scale of nearly 3 cluster radii. The data needed for this determination are now being taken.

\section{Discussion}

The basic equation, written for the 'slab' model (all variables uniform) is $R M=$ $n_{e} B L$, where the quantities are in the units defined on the first page, and I have dropped the numerical coefficient $(=0.81)$. This is sufficiently accurate for the purposes of estimation. What magnetic fields do these data suggest?

The origin of the RM is clearly in the cluster, and not from within the radio lobes, since the fit of position angle to $\lambda^{2}$ is perfectly linear over a range of more than 3 full turns in position angle (for $3 \mathrm{C} 295$ ). What remains to be understoo od ${ }_{i}$ is whether there is a significant local origin of all or part of the RM. If the magnetic fields are distributed throughout the cluster, with perhaps a dependency on the cluster gas density, the derived magnetic fields are $\sim 2 \sqrt{N} \mu \mathrm{G}$, where $N$ is the number of cells along the line of sight. Arguments based on galactic wakes indicate $N \sim 10$ to 25 . This implies magnetic fields up to $10 \mu G$ for Cygnus A, and perhaps 3 times greater for $3 \mathrm{C} 295$. But a problem with this picture, in which the cells must be assumed to be uniformly distributed throughout the cluster, is that the resulting RM image should be uniformly 'patchy', and shouldn't show large-scale uniformity. Furthermore, why should 3C295 have so much larger an RM, when the X-ray and radio luminosities are so similar to Cygnus A's? Noting that the lower-power radio sources of Owen and Ge generally provide lower RMs, and that the extreme RMs shown here are all associated with high luminosity, or perhaps more relevantly, high density of the local environment, is it possible that all or part of the RMs are due to a local effect?

Considerations such as these lead to the hypothesis that the significant magnetic fields are concentrated near the surface of the radio source. One picture is that of a supersonically advancing radio lobe, driving a shock wave into the surrounding ICM. The shock enhances both the gas density and magnetic fields, enhancing the measured RM. Carilli et al. (1988) have found strong evidence that perhaps $1000 \mathrm{rad} \mathrm{m}^{-2}$ are due to this effect. A simple analysis indicates the magnetic field in the sheath is 15 to $50 \mu \mathrm{G}$, leading to fields in the undisturbed medium close 
to the radio source and within the cluster core radius of 7 to $10 \mu \mathrm{G}$. This in turn argues for a strong radial dependence of the cluster magnetic field, since so much of the oberved RM orginates near the surface of the radio source. I believe that many of the localized features may in fact be ascribed to this mechanism. Note especially the curved RM arc centered on radio knot ' $B$ ', shown by Carilli et al.. However, this model has trouble explaining the continuing high RM noted in the inner regions of the Western lobe of Cygnus A, a region where the radio lobe is at rest with respect to the ICM, but where the RMs are still very high. Furthermore, Hydra $\mathrm{A}$ is a type I radio source, which is likely to be statically confined by the ICM, where no supersonic shocks are expected in the external gas, but where the $\mathrm{RMs}$ are just as high as in Cygnus $\mathrm{A}$.

Geoff Bicknell, in a recent preprint, has given another explanation of the high RMs. His model incorporates the high magnetic fields of the radio source interiors with the high gas density of the exterior to account for all the RM in a surface mixing layer. The main challenge to him posed by this model is how to mix the two without destroying the large-scale order of the field needed to obtain the high RMs. Geoff discusses his model in more detail elsewhere in these proceedings.

\section{Summary}

I summarize thusly:

- The RMs of isolated radio galaxies are low, rarely exceeding $50 \mathrm{rad} \mathrm{m}^{-2}$.

- The RMs of lower powered radio galaxies in clusters range from hundreds to thousands of $\mathrm{rad} \mathrm{m}^{-2}$. Some or all of the larger values may be associated with the galaxies.

- High power radio galaxies in clusters have $\mathrm{RMs}$ exceeding $2000 \mathrm{rad} \mathrm{m}^{-2}$, with large scale ordering present, but also with gradients on all scales down to the kpc scale, at least.

- Internal depolarization has not been detected in any of these objects, leading to upper limits on the internal gas density of $\sim 10^{-4} \mathrm{~cm}^{-3}$.

- Cluster field intensities exceed $1 \mu \mathrm{G}$, and are likely greater than $10 \mu \mathrm{G}$.

- There is good evidence that a significant fraction of the RMs are due to a skin effect, but I doubt all the observed RM in these objects have such an origin.

\section{References}

Aizu, K., Inoue, M., Tabara, H., and Kato, T., 1989, These Proceedings Carilli, C.L., Perley, R.A., and Dreher, J.W., 1988, Ap.J., 334, L73.

Dreher, J.W., Carilli, C.L., and Perley, R.A., 1987, Ap.J., 316, 611.

Kato, T., Tabara, H., Inoue, M., and Aizu, K., 1987, Nature, 329,223.

Taylor, G.B., Perley, R.A., Inoue, M., Kato, T., Tabara, H., and Aizu, K., 1989, Preprint. 